\title{
Expression of Bacterial Superantigen Genes in Mice Induces Localized Mononuclear Cell Inflammatory Responses
}

\author{
Steven W. Dow* and Terry A. Potter ${ }^{\star \ddagger}$ \\ *Division of Basic Immunology, Department of Medicine, National Jewish Center for Immunology and Respiratory Medicine, \\ and ${ }^{\ddagger}$ The Cancer Center, University of Colorado Health Sciences Center, Denver, Colorado 80206
}

\begin{abstract}
Bacterial superantigens are potent $\mathrm{T}$ cell activators, and superantigen proteins have been injected into mice and other animals to study $T$ cell responses in vivo. When superantigen proteins are injected, however, the $\mathrm{T}$ cell stimulatory effects cannot be confined to specific tissues. Therefore, to target superantigen expression to specific tissues, we used gene transfer techniques to express bacterial superantigen genes in mammalian cells in vitro and in tissues in vivo. Murine, human, and canine cells transfected with superantigen genes in vitro all produced superantigen proteins both intracellularly and extracellularly, as assessed by bioassay, immunocytochemistry, and antigen ELISA. Superantigens produced by transfected eukaryotic cells retained their biologic specificity for $\mathrm{T}$ cell receptor binding. Intramuscular injection of superantigen plasmid DNA in vivo induced an intense intramuscular mononuclear cell infiltrate, an effect that could not be reproduced by intramuscular injection of superantigen protein. Intradermal and intravenous injection of superantigen DNA induced cutaneous and intrapulmonary mononuclear cell inflammatory responses, respectively. Thus, superantigen genes can be expressed by mammalian cells in vivo. Superantigen gene therapy represents a novel method of targeting localized $\mathrm{T}$ cell inflammatory reactions, with potential application to treatment of cancer and certain infectious diseases. (J. Clin. Invest. 1997. 99: 2616-2624.) Key words: SEA $\bullet$ T cells $\bullet$ muscle $\bullet$ lung $\bullet$ skin
\end{abstract}

\section{Introduction}

Certain bacterial proteins, designated superantigens (SAgs), possess the ability to stimulate $\mathrm{T}$ cell proliferation and cytokine secretion in a process that requires cross-linking of $\mathrm{T}$ cell receptor (TCR) and MHC class II molecules (1-5). Staphylo-

Address correspondence to Dr. Terry A. Potter, Department of Medicine, National Jewish Center for Immunology and Respiratory Medicine, 1400 Jackson Street, Denver, CO 80206. Phone: 303-398-1311; FAX: 303-398-1396.

Received for publication 8 October 1996 and accepted in revised form 30 January 1997.

1. Abbreviations used in this paper: APC, antigen-presenting cell; CHO, Chinese hamster ovary; SAg, superantigen; SEA, staphylococcal enterotoxin A; SEB, staphylococcal enterotoxin B; TCR, T cell receptor; TSST-1, toxic shock syndrome toxin $1 ; \mathrm{V} \beta$, T cell receptor variable region beta chain.

J. Clin. Invest.

(C) The American Society for Clinical Investigation, Inc. 0021-9738/97/06/2616/09 \$2.00

Volume 99, Number 11, June 1997, 2616-2624 cocci produce numerous SAgs, many of which were first identified by their enterotoxigenic properties, including staphylococcal enterotoxins A-E, G, H, and toxic shock syndrome toxin (TSST-1). Other SAgs have been identified from streptococci, Pseudomonas, and mycoplasmas $(3,5)$. Superantigens stimulate an oligoclonal population of T cells, based on SAg binding to specific $T$ cell receptor variable region $\beta$ chains, a property that distinguishes SAgs from conventional $\mathrm{T}$ cell mitogens (6-8). Superantigens also bind with high affinity to MHC class II molecules (9-14), and the crystal structure of staphylococcal enterotoxin B (SEB) complexed to HLA-DR1 molecule has been reported (8). The site of SAg-MHC class II interaction is distinct from the region of the $\mathrm{MHC}$ molecule that binds antigenic peptides, and the presence of MHC class II positive accessory cells is required for optimal $\mathrm{T}$ cell activation by SAgs $(6,15-20)$. Superantigens are able to activate both $\mathrm{CD}^{+}$and $\mathrm{CD}^{+}{ }^{+} \mathrm{T}$ cells via TCR cross-linking to $\mathrm{MHC}$ class II molecules.

The biologic properties of SAgs make them attractive for use in immunotherapy. Superantigens administered systemically to mice induce $\mathrm{T}$ cell activation, followed by either deletion or prolonged anergy. Accordingly, administration of high doses of SAgs (e.g., SEB) has been used to reduce the severity of several experimental oligoclonal $\mathrm{T}$ cell-mediated diseases, including experimental allergic encephalitis (21), spontaneous diabetes mellitus (22), and allergic hypersensitivity (23). Paradoxically, others have found that bacterial SAgs may actually exacerbate certain experimental immune-mediated diseases such as adjuvant arthritis (24) and allergic encephalitis (25). T cells stimulated by SAgs proliferate and release primarily Th1 cytokines (especially interferon-gamma and TNF alpha), and the $\mathrm{T}$ cell stimulatory properties of SAgs have also been used successfully to treat experimental cancer in mice (26-28).

These studies have demonstrated the potential application of SAgs to disease models in rodents, but humans and other mammals are much more susceptible to the toxic effects of SAgs than are rodents $(5,6,29)$. In humans, SAgs released by bacteria at localized sites of infection are capable of inducing significant morbidity and mortality, including toxic shock syndrome, an often fatal disease associated with TSST-1-producing strains of staphylococci $(5,29)$. Furthermore, administration of even small quantities $(\mu \mathrm{g})$ of purified SAgs to mammals induces massive $\mathrm{T}$ cell cytokine release, and reproduces many of the signs associated with toxic shock syndrome $(30,31)$.

For SAgs to be useful therapeutically, their potential toxicity must be minimized. We therefore investigated a genetic approach to delivering sustained, locally high concentrations of bacterial SAgs. Three different bacterial SAg genes (SEB, staphylococcal enterotoxin A [SEA], and TSST-1) were cloned into eukaryotic expression plasmids. Mammalian cells transfected with these plasmids produced SAg proteins (as assessed by bioassays, antigen-capture ELISA, and immunocytochemistry) and the SAgs produced by transfected cells retained 
their biologic TCR specificity. In vivo, direct SAg plasmid DNA injection into skeletal muscles and skin of mice induced a pronounced and rapid mononuclear cell inflammatory response. In addition, intravenous injection of SAg DNA-lipid complexes induced an intrapulmonary perivascular inflammatory response. These data indicate that bacterial SAgs can be expressed in mammalian cells using appropriate expression vectors, and suggest the feasibility of targeted in vivo gene therapy with SAgs.

\section{Methods}

Animals. B10.BR mice were purchased from The Jackson Laboratory (Bar Harbor, ME). Female mice between 12 and 20 wk of age were used for experiments.

Reagents. Recombinant SEB and SEA and affinity-purified rabbit and sheep antisera to SEB and SEA were purchased from Toxin Technologies (Sarasota, FL). Lipofectamine was purchased from GIBCO-BRL (Gaithersburg, MD).

Cells and cell lines. A CD4+, T cell reseptor variable region beta chain $(\mathrm{V} \beta) 3+\mathrm{T}$ cell hybridoma $(5 \mathrm{KC})$, and a $\mathrm{CD} 4+, \mathrm{V} \beta 3+\mathrm{T}$ cell clone (AD10) were obtained from Dr. Philippa Marrack (National Jewish Hospital, Denver, CO) and Dr. Steve Hedrick (University of California, San Diego), respectively. Normal human leukocytes were obtained from healthy human donors, and the peripheral blood mononuclear cells (PBMC) were separated by Ficoll-Hypaque density gradient centrifugation. The B16 murine melanoma cell line was obtained from Dr. Isiah Fidler (M.D. Anderson, Houston, TX). The human melanoma cell line SK38 was obtained from Dr. Pat Walsh (School of Medicine, University of Colorado). Canine melanoma cell lines were obtained from biopsy specimens from dogs with spontaneous oral malignant melanoma (Dow, S.W., unpublished data). Cells were maintained in modified Eagle's medium supplemented with 5\% fetal bovine serum.

Superantigen expression vectors and in vitro transfections. The genes for SEA and SEB were obtained from Dr. John Kappler (National Jewish Hospital), and the gene for TSST-1 was obtained from Dr. Brian Kotzin (National Jewish Hospital). After PCR amplification, all three genes were subcloned into a eukaryotic expression vector (PCR3; Invitrogen Corp., San Diego, CA). The primers used for cloning SEA were (forward): GGGAATTCCATGGAGAGTCAACCAG; (backward): GCAAGCTTAACTTGTTAATAG; for SEB(forward): GGGAATTCCATGGAGAAAAGCG; (backward): GCGGATCCTCACTTTTTCTTTG; for TSST-1 (forward): CGGGGTACCCCGAAGGAGGAAAAAAAAATGTCTACAAACGATAATATAAAG; (backward): TGCTCTAGAGCATTAATTAATTTCTGCTTCTATAGTTTTTAT. The full-length TSST-1 gene was cloned into PCR3, whereas only the mature SEB and SEA genes (minus the putative bacterial signal sequences) were cloned into PCR3. Removal of the SEB and SEA signal sequences increased the level of gene expression in transfected cells (data not shown). The plasmids were grown in Escherichia coli, and plasmid DNA was extracted by the modified alkaline lysis method and purified on a $\mathrm{CsCl}$ gradient. Complexes of plasmid DNA with cationic lipids for intravenous injection were prepared by the Megabios Corporation (Burlingame, CA). Briefly, the lipids for intravenous DNA delivery were comprised of DOTIM (octadecenoyloxy[ethyl-2-heptadecenyl-3 hydroxyethyl]imidazolinium chloride) and cholesterol in a 1:1 molar ratio, and were complexed to plasmid DNA in a 1:6 ratio ( $\mu$ g DNA to nmol lipid) as described previously (32). This lipid formulation has been shown to induce superior pulmonary delivery and expression of a plasmidencoded reporter gene in mice after intravenous injection (32).

In vitro cell transfections were done in 12-well plates, using $3.0 \mu \mathrm{g}$ plasmid DNA and Lipofectamine (GIBCO BRL), at $37^{\circ} \mathrm{C}$ for $4 \mathrm{~h}$. After transfection, the cells were cultured in $2.0 \mathrm{ml}$ complete medium for $48 \mathrm{~h}$, and the supernatants were harvested. The cells were then washed in PBS, and cell lysates were prepared by three alternating freeze-thaw cycles in $2.0 \mathrm{ml}$ of medium, followed by centrifugation to remove cell debris. Stably transfected Chinese hamster ovary $(\mathrm{CHO})$ and B16 lines were isolated by selection in $1.0 \mathrm{mg} / \mathrm{ml} \mathrm{G} 418$ (GIBCO BRL). Cells were grown and passaged in medium containing G418 for 3-4 wk. Mock transfected cell lines (transfected with empty $\mathrm{PCR}_{3}$ vector and selected in G418) were used as controls.

Assays for SAg biologic activity. Supernatants and lysates from SAg-transfected cells were assayed for biologic activity by measuring the proliferative response of human PBMC, B10.BR splenocytes, or $\mathrm{T}$ cell clones. Assays were done in flat-bottomed microtiter plates in modified Eagle's medium supplemented with $10 \%$ FCS, 2-mercaptoethanol $\left(5 \times 10^{-5} \mathrm{M}\right)$, glutamine, penicillin, and streptomycin. $100 \mu \mathrm{l}$ of a $5 \times 10^{6} / \mathrm{ml}$ suspension of spleen cells or human PBMC was added to triplicate wells in which $100 \mu$ l of test supernatants or lysates had been serially diluted. Cells were incubated for $3 \mathrm{~d}$ at $37^{\circ} \mathrm{C}$, then pulsed with $1.0 \mu \mathrm{Ci}\left[{ }^{3} \mathrm{H}\right]$ thymidine/well, and harvested $18 \mathrm{~h}$ later. Incorporated $\left[{ }^{3} \mathrm{H}\right]$ activity was quantitated on an automated beta counter (Wallac, Gaithersburg, MD).

The proliferative response of the $\mathrm{V} \beta 3+\mathrm{T}$ cell clone AD10 to SEA produced by transfected B16 cells was quantitated by coculture of $5 \times 10^{4} \mathrm{AD} 10$ cells, $5 \times 10^{5}$ irradiated $(3,000 \mathrm{rad})$ syngeneic spleen cells, and $2 \times 10^{4}$ irradiated (12,000 rad) B16 cells transfected with the SEA plasmid, or mock-transfected with vector only. Proliferation was quantitated after $2 \mathrm{~d}$ in culture as described above. Recombinant SEA $(10 \mathrm{ng} / \mathrm{ml})$ was used as a positive control. The ability of supernatants from SAg-transfected cells to stimulate IL-2 production by a hybridoma expressing a V $33+$ TCR was assessed using the $5 \mathrm{KC}$ hybridoma and an HT-2 bioassay for IL-2 activity (33).

$S E B$ antigen ELISA. The concentration of SEB in lysates and supernatants of transfected cells was assayed using an antigen-capture ELISA. Assay plates (Dynatech Laboratories, Inc., Chantilly, VA) were coated with $10.0 \mu \mathrm{g} / \mathrm{ml}$ monoclonal anti-SEB antibody (B344.1, provided by Dr. John Kappler, National Jewish Hospital [34]), then blocked with $3 \%$ BSA for $2 \mathrm{~h}$. The plates were incubated with test samples for $2 \mathrm{~h}$ at room temperature, then washed and incubated with a 1:5,000 dilution of biotinylated sheep anti-SEB (Toxin Technologies). Plates were washed again and incubated with streptavidin-HRP (Zymed Labs, Inc., S. San Francisco, CA), then the substrate was developed with ABTS solution (Bio-Rad Laboratories, Richmond, CA). SEB concentrations in test samples were determined by comparison to a standard curve generated with known concentrations of recombinant SEB. This ELISA was sensitive, with a lower limit of detection of $1.0 \mathrm{pg} / \mathrm{ml} \mathrm{SEB}$, and was linear up to $100 \mathrm{ng} / \mathrm{ml} \mathrm{SEB}$, and did not cross-react with other SAgs, including SEA and TSST-1 (data not shown).

Immunocytologic detection of SAg proteins in in vitro transfected cells. Immunoreactive SEB and SEA protein in transfected $\mathrm{CHO}$ cells was detected using affinity-purified rabbit antiserum to either SEB or SEA (Toxin Technologies). Cells were grown in culture slides (Lab-Tek, Naperville, IL), transfected with either SEA, SEB, or empty vector DNA using Lipofectamine, then cultured for $48 \mathrm{~h}$. Cells were then fixed in acetone-methanol, and incubated sequentially with a 1:1,500 dilution of primary antiserum, biotinylated goat antirabbit IgG antiserum (Kirkegaard and Perry Laboratories, Gaithersburg, MD), and streptavidin-HRP (Zymed Labs). Aminoethylcarbazole was used as a substrate, and cells were lightly counterstained with hematoxylin and coverslipped for photography. Controls included mock-transfected cells incubated with anti-SEB or -SEA antiserum, and SEB and SEA transfected cells incubated with irrelevant rabbit antiserum.

In vivo DNA injections: intramuscular, intradermal, intravenous. Plasmid DNA for intramuscular injection was diluted to a final concentration of $0.5 \mathrm{mg} / \mathrm{ml}$ in sterile PBS. Mice were anesthetized, and both quadriceps muscles were injected in two sites, each with a total of $100 \mu \mathrm{l}$ of DNA PBS solution per muscle. Mice were injected with plasmid DNAs encoding either SEA, SEB, or TSST-1, or with an equivalent amount of empty vector DNA as a control for nonspecific responses to DNA injection. Plasmid DNA encoding a conventional 
antigen (ovalbumin cDNA; provided by Dr. Michael Bevan, University of Washington, Seattle, WA) was also used in some experiments. Other mice were injected intramuscularly with $1.0 \mu \mathrm{g}$ SEA protein diluted in $100 \mu \mathrm{l}$ PBS.

Intradermal injections were done with $50 \mu \mathrm{g}$ plasmid DNA, encoding either SEA, or empty vector in a total volume of $100 \mu \mathrm{l}$, which was injected intradermally into the flank skin of anesthetized mice. Intravenous delivery of SAg genes was accomplished by injecting plasmid DNA complexed to DOTIM-cholesterol lipid (32). $120 \mu \mathrm{g}$ of SEA or empty vector DNA in a total volume of $200 \mu \mathrm{l}$ was injected once intravenously.

Tissue histology. Muscle tissues from DNA-injected mice were examined histologically to assess the effect of local gene expression on cellular infiltration. 4-14 d after DNA injection, mice were killed, and quadriceps muscle tissues were removed. Skin and lung tissues were removed at $6 \mathrm{~d}$ after injection. In mice injected intravenously, lung tissues were perfused with PBS plus heparin, and tissues from lungs and all major organs were harvested. Tissues were fixed in formalin for routine histologic evaluation. Formalin-fixed tissues were sectioned to a thickness of $4.0 \mu \mathrm{m}$, and were stained with hematoxylin and eosin.

Immunohistology. Tissues were harvested as described above, and snap frozen in OCT compound (Miles Laboratories Inc., Naperville, IL). Specimens were cryosectioned to a thickness of $4.0 \mu \mathrm{g}$, then fixed in acetone. After blocking nonspecific binding and quenching endogenous peroxidase activity, the specimens were incubated with rat mAbs to either CD4 (clone R4-5; PharMingen, San Diego, CA), CD8, alpha chain (clone 53.6.7), or Mac-1 (clone M1/70). After washing, specimens were incubated sequentially with biotinylated donkey anti-rat antisera (Jackson ImmunoResearch Labs, Inc., West Grove, PA), avidin-biotin complexes (Elite kit; Vector Laboratories, Inc., Burlingame, CA), then developed with diaminobenzidine, counterstained lightly with hematoxylin, and coverslipped. Controls included use of an irrelevant rat $\mathrm{mAb}$ and omission of the primary antibody.

\section{Results}

Eukaryotic cells express SAg biologic activity when transfected with SAg genes. Both supernatants and lysates from $\mathrm{CHO}$ cells transfected with eukaryotic expression plasmids encoding
SEB, SEA, or TSST-1, stimulated strong lymphocyte proliferation in human PBMCs, compared to $\mathrm{CHO}$ cells transfected with empty vector DNA (Fig. $1 A$ ) For all three SAgs, supernatants from $\mathrm{CHO}$ cells stimulated greater lymphocyte proliferative activity than cell lysates. Supernatants and lysates from SEB and SEA (Fig. $1 B$ ) or TSST-1 (data not shown) transfected $\mathrm{CHO}$ cells also stimulated proliferation of mouse spleen cells. The stimulatory activity of SEB was generally lower than that of SEA in mouse spleen cell assays.

Supernatants and lysates from SAg-transfected human melanoma cells also stimulated proliferation of human PBMC (Fig. $1 C$ ), but unlike the case with $\mathrm{CHO}$ cells, lysates from human melanoma cells had greater $\mathrm{T}$ cell stimulatory activity than did supernatants. Murine and canine melanoma cells transfected with SAgs in vitro also produced strong lymphocyte stimulatory activity (data not shown), indicating that multiple different species and cell types were capable of producing biologically active SAgs after transfection. These data demonstrated that bacterial SAg genes expressed under the control of eukaryotic promoters in mammalian cells produced biologically active SAg proteins, both intracellularly and extracellularly.

SAgs are secreted by stably transfected cells. The preceding experiments suggested that SAg proteins were released from transfected cells, in addition to being produced intracellularly. In transient transfection experiments, however, it was not possible to distinguish whether SAg proteins were being released from dead and dying cells or from intact cells. We therefore established stable SAg-transfected lines to study the kinetics of SAg production and release from viable cells. Lymphocyte stimulatory activity in serially sampled supernatants harvested from SEA- and SEB-transfected $\mathrm{CHO}$ cell lines increased progressively with time (Fig. 2). Thus, SAgs were released constituitively from intact transfected cells, either by simple diffusion across a concentration gradient, or by an active transport process.

Immunocytochemical detection of intracytoplasmic SAgs within transfected cells. Immunocytochemistry was used to di-
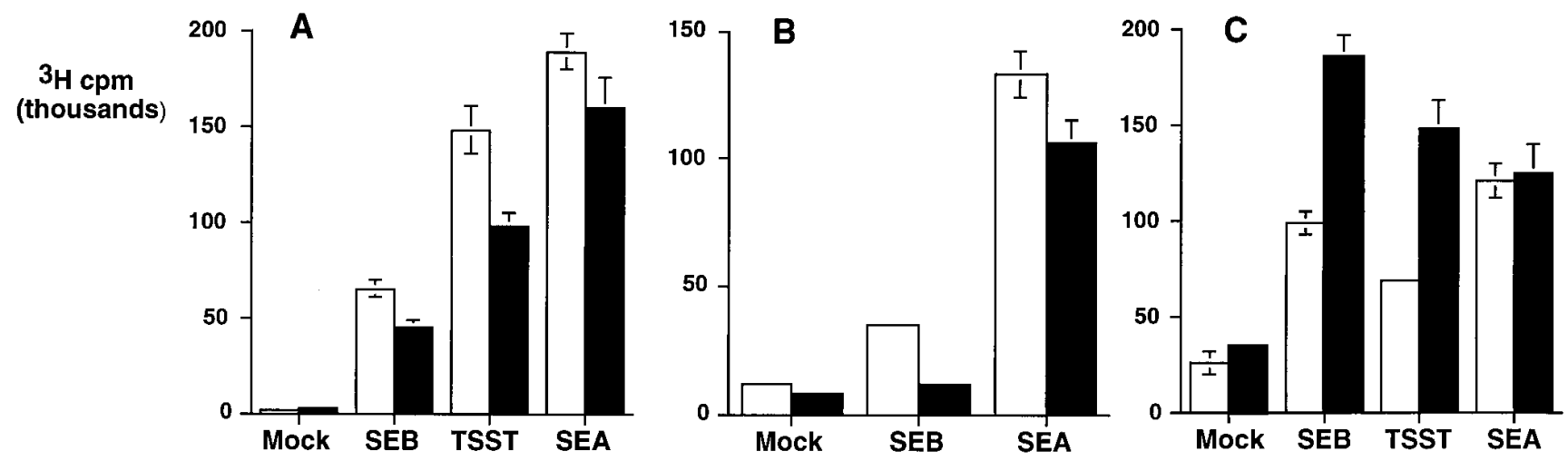

Figure 1. Supernatants and lysates from cells transfected with SAg genes stimulate proliferation of human and mouse T cells. T cell proliferation was used to assay the biologic activity of SAg genes expressed in eukaryotic cells. CHO $(A$ and $B)$ or a human melanoma cell line (SK28; $C)$ was transfected with $3.0 \mu \mathrm{g}$ plasmid DNA encoding either SEB, SEA, TSST-1, or empty vector (Mock) for $4 \mathrm{~h}$ at $37^{\circ} \mathrm{C}$, using Lipofectamine. Supernatants and lysates were assayed in quadruplicate for lymphocyte stimulatory activity, using normal human PBMC $(A$ and $C)$ or mouse spleen cells $(B)$, as described in Methods. Supernatants (open bars) or lysates (filled bars) from CHO cells transfected with the genes for SEB, TSST-1, or SEA stimulated proliferation of human PBMC, compared to mock transfected controls $(A)$. Splenocytes from B10.BR mice proliferated when cultured with lysates (filled bars) or supernatants (open bars) from SEB- or SEA-transfected CHO cells, compared to mock transfected controls $(B)$. Lysates (filled bars) from human melanoma cells transfected with SAg genes stimulated greater proliferation of human PBMC than did supernatants (open bars) $(C)$. Error bars represent SD. 
SEB

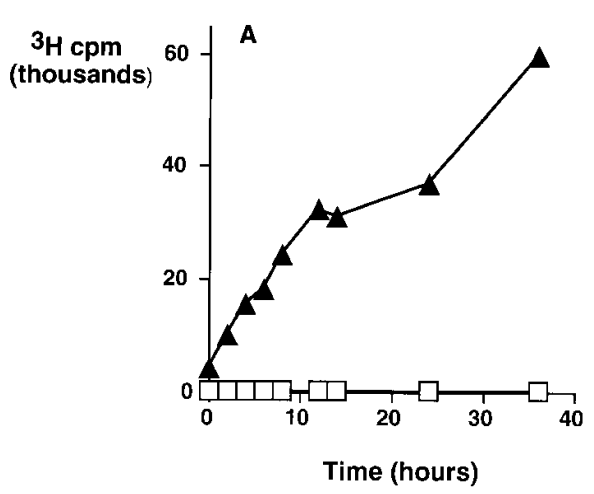

SEA

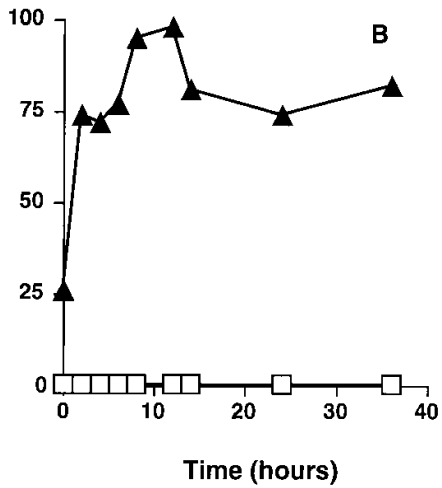

Figure 2. SEB and SEA are secreted by stably transfected cells. Stably transfected CHO cells were used to detect secretion of biologically active SAg proteins. Stably transfected $\mathrm{CHO}$ cells were seeded into nine separate wells of a 24-well plate, adhered overnight, then washed with PBS, and $1.0 \mathrm{ml}$ fresh medium was added. Supernatants were then harvested from one well at each time point, and frozen before assay for SAg biologic activity. Supernatants were assayed for T cell stimulatory activity, using normal human PBMC, as described in Fig. 1 and Methods. The PBMC stimulatory activity present in serially harvested supernatants from mock-transfected $\mathrm{CHO}$ (open squares) versus SEB $(A)$ (closed triangles) or SEA-transfected $\mathrm{CHO}$ cells $(B)$ was quantitated. For both SEB- and SEA-transfected CHO cell lines, $\mathrm{T}$ cell stimulatory activity in supernatants of the transfectants increased with time of culture, consistent with SAg secretion by stably transfected cells. rectly demonstrate $\mathrm{SAg}$ protein production in in vitro transfected cells. Strong positive staining for immunoreactive SEB and SEA proteins was observed intracellularly in 48-h transfected CHO cells (Fig. $3 a$ and $b$ ), compared to mock-transfected cells (Fig. $3 d$ ). Superantigen expression could be detected beginning as early as $12 \mathrm{~h}$ after transfection (not shown). Although transiently transfected cells produced substantial amounts of intracellular SAg protein, stably transfected $\mathrm{CHO}$ cells expressed much lower levels (Fig. $3 \mathrm{c}$ ). Thus, prolonged exposure to high intracellular SAg concentrations may be lethal to some cells, such that only low levels of intracellular SAg expression can be tolerated for prolonged periods.

Quantitation of intracellular and extracellular SEB production by transfected $\mathrm{CHO}$ cells. An antigen ELISA was used to quantitate SEB protein production by transfected $\mathrm{CHO}$ cells. Serial dilutions of supernatants and lysates from 48-h transfected $\mathrm{CHO}$ cells $\left(5 \times 10^{5}\right.$ cells $)$ were assayed in triplicate, using an SEB-specific antigen ELISA. Cells were transfected with either empty vector, SEB, or SEA. The concentration of
SEB was determined by comparison to a standard curve generated with recombinant SEB. Results are representative of three separate experiments. Supernatants contained $13.0 \mathrm{pg} / \mathrm{ml}$ SEB, whereas lysates contained $3.0 \mathrm{pg} / \mathrm{ml} \mathrm{SEB}$ (Table I). By immunocytochemistry, $10 \%$ of cells $\left(5 \times 10^{4}\right.$ cells $)$ expressed detectable SEB protein (see Fig. 3). Thus, it was estimated that an individual high-expressing cell produced $\sim 0.12 \mathrm{fg}$ of intracellular SEB and $0.52 \mathrm{fg}$ of extracellular SEB, assuming that all the intracellular SAg protein was completely liberated by freeze thawing. Depending on the efficiency of gene transfer, these data suggest that in vivo transfected cells could therefore serve as a significant source of local SAg activity in vivo, inasmuch as $T$ cells can respond to concentrations of SEB or SEA as low as 1.0-10.0 fg/ml (20).

Supernatants from SEA-transfected cells stimulate $T$ cell proliferation in a V $\beta$-specific manner. Superantigens differ from conventional lymphocyte mitogens in that they only bind and stimulate $\mathrm{T}$ cells expressing certain TCR V $\beta$ chains, whereas mitogens such as concanavalin A stimulate $\mathrm{T}$ cells irrespective

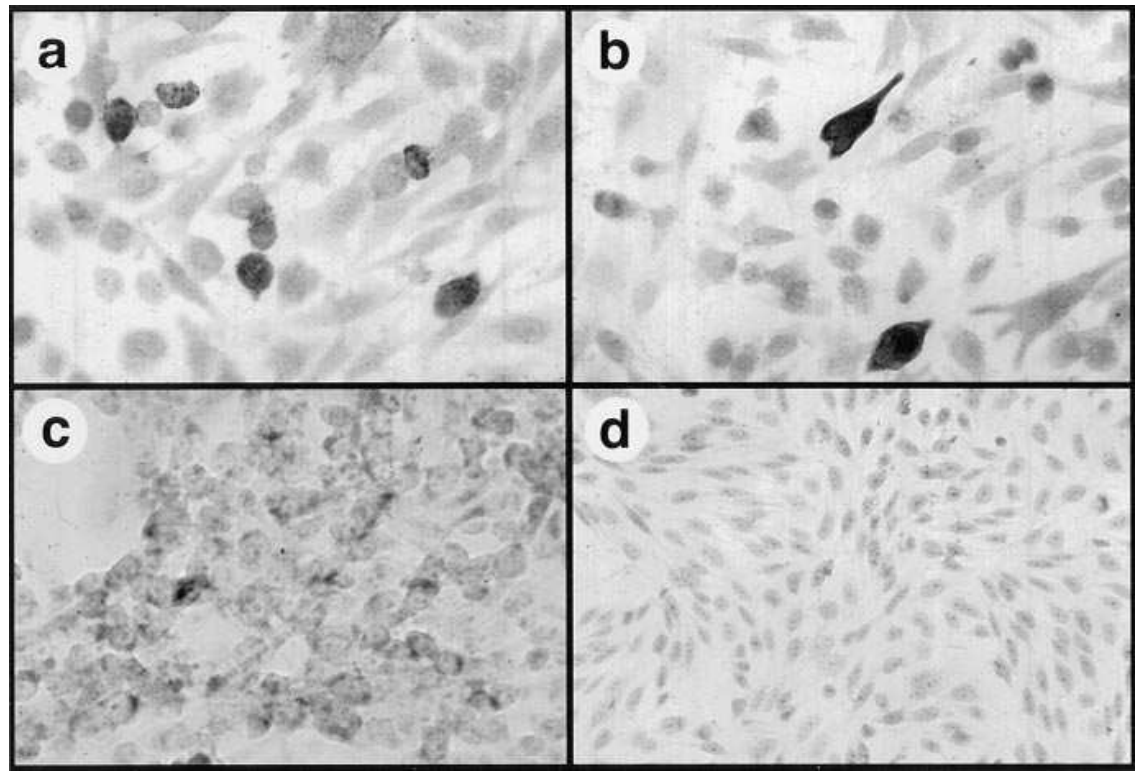

Figure 3. Transfected cells express high intracellular concentrations of SEB and SEA. SEB and SEA-specific antisera were used to localize intracellular SAg proteins in transfected cells in vitro. CHO cells were fixed $48 \mathrm{~h}$ after transfection, and immunostained using rabbit anti-SEB or anti-SEA antiserum, as described in Methods. CHO cells transfected with SEB $(a)$ or with SEA $(b)$ expressed high levels of intracytoplasmic SAg proteins. Mocktransfected CHO cells did not stain with SEB antiserum $(d)$, nor did SEB- or SEA-transfected cells stain with an irrelevant rabbit antiserum (data not shown). $\mathrm{CHO}$ cells selected for stable SEB expression (c) expressed much lower levels of intracellular SEB protein than did transiently transfected cells $(b)$. $\times 170$. 
Table I. Quantitation of SEB Production by In Vitro Transfected CHO Cells

\begin{tabular}{llc}
\hline Gene transfected & \multicolumn{1}{c}{ Assayed } & SEB $(\mathrm{pg} / \mathrm{ml})^{*}$ \\
\hline SEB & Supernatant & 13.0 \\
SEB & Lysate & 3.0 \\
SEA & Supernatant & 0 \\
SEA & Lysate & 0 \\
Empty vector & Supernatant & 0 \\
Empty vector & Lysate & 0
\end{tabular}

*Values are mean SEB concentration from triplicate wells of transfected CHO cells.

of which TCR V $\beta$ they express. In mice, for example, SEA activates $\mathrm{T}$ cells that express $\mathrm{V} \beta 1,3,10,11,12$, and 17 TCRs, whereas SEB activates T cells that express the TCR V 7 and 8.1-8.3 chains (3). The TCR V $\beta$ specificity of SAgs was therefore used to demonstrate that the lymphocyte stimulatory activity present in supernatants of SAg-transfected CHO cells was not due to a nonspecific mitogenic effect. Irradiated, SEAtransfected B16 cells stimulated proliferation of AD10 cells (a $\mathrm{V} \beta 3+, \mathrm{CD} 4+\mathrm{T}$ cell clone) in the presence of syngeneic splenic antigen-presenting cells (APC) (Fig. $4 A$ ). In the absence of APCs, or in the presence of mock-transfected B16 cells, the AD10 cells did not proliferate (Fig. $4 A$ ).

Supernatants and lysates from SEA-transfected $\mathrm{CHO}$ cells also stimulated IL-2 production by the $\mathrm{V} \beta 3+\mathrm{T}$ cell hybridoma 5KC (Fig. 4 B). Supernatants or lysates from SEB-transfected or mock-transfected $\mathrm{CHO}$ cells did not stimulate IL-2 production (Fig. $4 \mathrm{~B}$ ). Thus, the T cell stimulatory properties of proteins produced by mammalian cells transfected with bacterial SAgs were consistent with those of the recombinant SAg molecules, indicating that the SAg produced by transfected mammalian cells was functional and TCR V $\beta$ specific.

Intramuscular injection of SAg genes induces a marked mononuclear cell inflammatory response. Plasmid DNAs injected directly into muscle tissue are efficiently expressed, leading to immune responses to proteins encoded by the in- jected genes (35). Therefore, we injected SAg plasmids into quadriceps muscles of B10.BR mice, and assessed the intramuscular inflammatory response histologically, as an indirect measure of in vivo gene expression. Injection of either empty vector plasmid DNA or an expression vector encoding the ovalbumin cDNA elicited only minimal inflammatory responses in injected muscle tissues 1 wk after injection (Fig. 5, $a$ and $b$ ). By contrast, injection of plasmid DNA encoding either SEA, SEB, or TSST-1 induced a marked mononuclear cell inflammatory response (Fig. 5, $d-f$ ). The mononuclear cell infiltrate was present between muscle fiber bundles, and also in loose connective tissues adjacent to muscle groups. The inflammatory infiltrate was not uniform within muscle tissues, but was instead distributed in a multifocal pattern. This inflammatory infiltrate distribution was consistent with the pattern of gene expression observed in muscle tissues after injection of a $\beta$-galactosidase reporter gene (data not shown). The inflammatory response induced by injection of SEA and TSST-1 DNA was generally stronger than that induced by SEB DNA injection. Intramuscular injection of $1.0 \mu \mathrm{g}$ recombinant SEA in PBS elicited only a mild inflammatory response, similar to that induced by injection of empty vector DNA (Fig. $5 \mathrm{c}$ ). Thus, prolonged SAg expression achieved by gene transfer may be necessary to induce strong local inflammatory responses in tissues such as skeletal muscle, whereas SAg proteins injected into such tissues do not persist long enough to induce significant inflammatory cell infiltration.

Sequential examination of SEA-injected muscle specimens revealed that mononuclear cell infiltration began by $48 \mathrm{~h}$ after DNA injection, reached a maximum at 5-6 d, then began to subside thereafter (data not shown). By 2 wk, the inflammatory response had diminished considerably, and consisted largely of monocytes and scattered foci of T cells. Thus, the local inflammatory effects of SAg gene expression were transient, most likely due to transient in vivo gene expression. The intramuscular inflammatory cell infiltrate was comprised primarily of two cell populations, based on immunohistochemical staining of muscle tissues (data not shown). Approximately $50 \%$ of the mononuclear cells were T cells (both CD4+ and $\mathrm{CD} 8+$ ), and the other $50 \%$ were Mac-1 positive monocytes and macrophages. Occasional polymorphonuclear cells (neu-
AD10

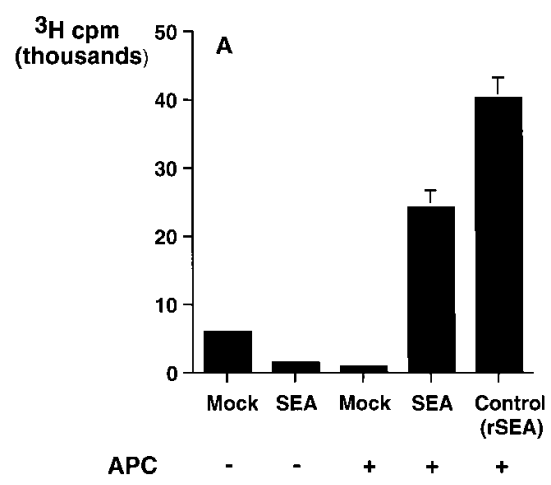

$5 \mathrm{KC}$

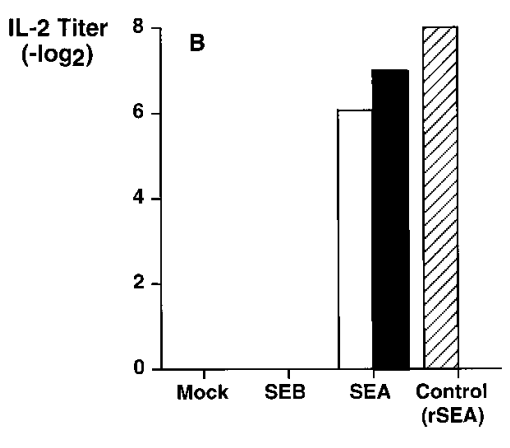

Figure 4. (A) SEA-transfected cells stimulate proliferation of $\mathrm{V} \beta 3+\mathrm{T}$ cells. To determine whether the $\mathrm{T}$ cell stimulatory activity generated by SAg-transfected cells was TCR V $\beta$-specific, we assessed the proliferative responses of a $\mathrm{V} \beta 3+\mathrm{T}$ cell clone (AD10) to coculture with SEA-transfected B16 melanoma cells. As a source of syngeneic APC, irradiated splenocytes from B10.BR mice were added to APC $(+)$ wells, while APC (-) wells received only B16 cells and AD10 cells. Recombinant SEA (100 ng/ml) was added to AD10 cells plus APCs as a positive control. After $2 \mathrm{~d}$ of incubation, proliferation was quantitated by $\left[{ }^{3} \mathrm{H}\right]$ thymidine incorporation, and the mean uptake was plotted along with SD. These data are representative of two separate experiments. (B) Supernatants and lysates from SEA-, but not SEB-

transfected cells stimulate IL-2 production by a V $\beta 3+\mathrm{T}$ cell hybridoma. A V $\beta 3+\mathrm{T}$ cell hybridoma (5KC) was used in a second assay for TCR specificity. Test supernatants from transfected CHO cells were added to $5 \mathrm{KC}$ cells, along with irradiated B10.BR splenocytes as APCs. After $24 \mathrm{~h}$ of incubation, supernatants were harvested and assayed for IL-2 activity, using HT- 2 cells. Data were plotted as the mean supernatant dilution that contained detectable IL-2 activity. Similar results were obtained in one additional experiment. 


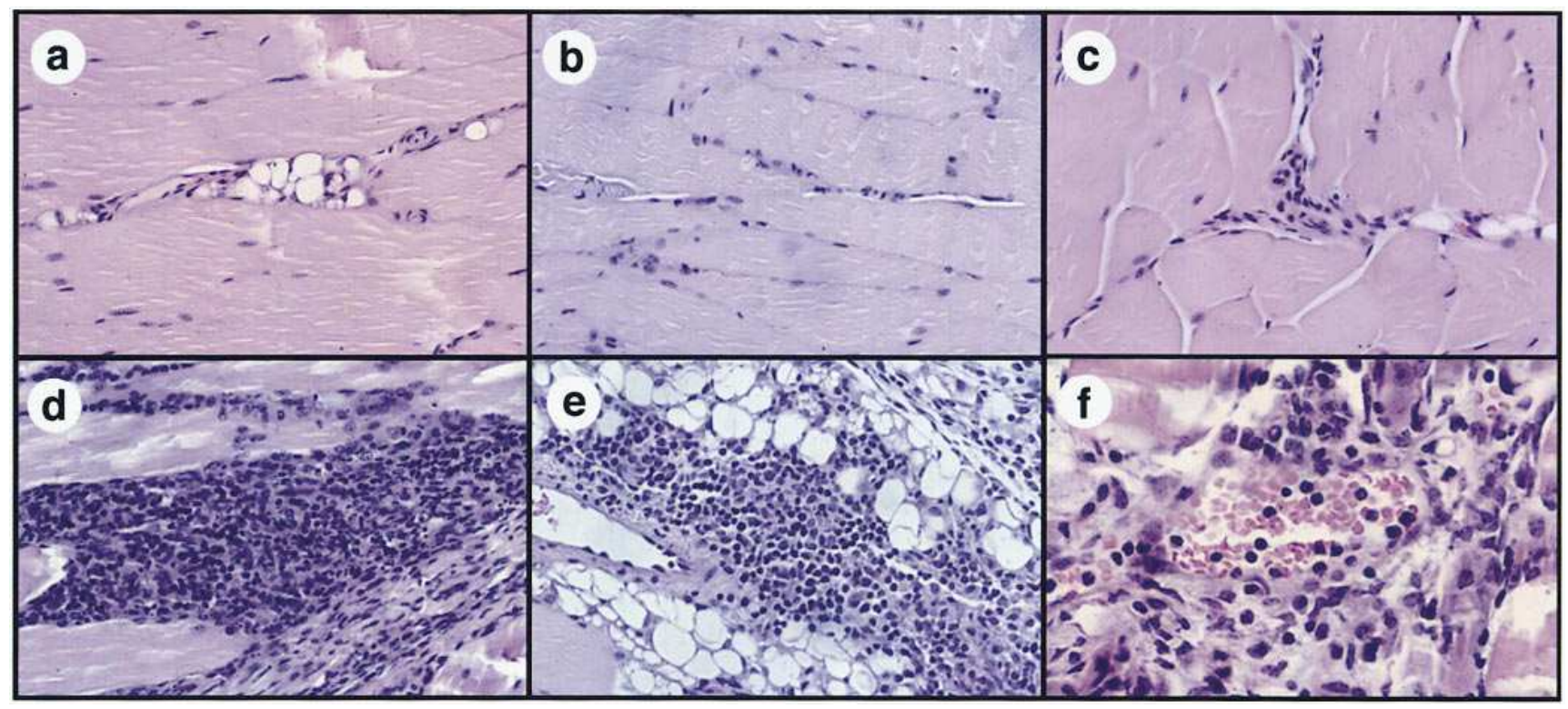

Figure 5. Intramuscular injection of SAg plasmid DNA induces a strong mononuclear cell inflammatory response. Quadriceps muscles of B10.BR mice were injected once with $50 \mu \mathrm{g}$ plasmid DNA encoding SEB, SEA, TSST-1, chicken ovalbumin, or empty vector DNA. The plasmid DNA was diluted to $0.5 \mathrm{mg} / \mathrm{ml}$ in PBS before injection. Other mice were injected once intramuscularly with $1.0 \mu \mathrm{g}$ recombinant SEA protein. $1 \mathrm{wk}$ later $(a-e)$ or $4 \mathrm{~d}$ later $(f)$, mice were killed and the quadriceps muscles were harvested, fixed in formalin, sectioned, and stained with hematoxylin and eosin. Intramuscular injection of empty vector plasmid DNA $(a)$ or a plasmid DNA encoding ovalbumin induced a minimal inflammatory response $(b)$, as did $1.0 \mu \mathrm{g}$ recombinant SEA protein $(c)$. By contrast, a marked mononuclear cell infiltrate developed in muscle tissues from mice injected with plasmid DNAs encoding either TSST $(d)$, SEB $(e)$, or SEA $(f)$. In occasional sections, particularly at the 4-d time point, mononuclear cells emigrating from intramuscular blood vessels into perivascular tissues were observed ( $f$ ). By 2 wk after injection, the inflammatory response had diminished considerably (data not shown). Similar results have been obtained in numerous SAg DNA-injected mice. $\times 170$ for $a-e$ and $\times 350$ for $f$.

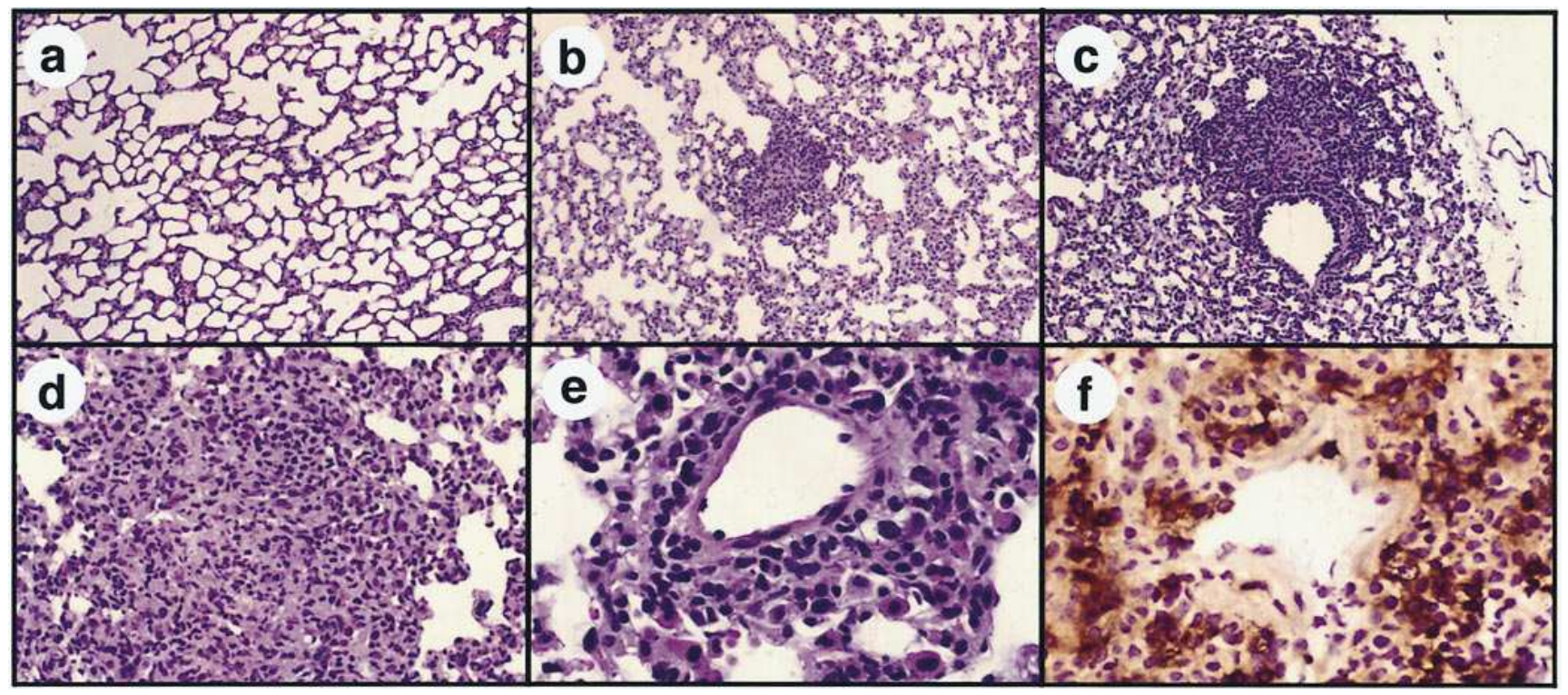

Figure 7. Intravenous injection of SEA DNA complexed to cationic lipids induces pulmonary perivascular inflammation. Mice (two per group) were injected once intravenously with $120 \mu \mathrm{g}$ plasmid DNA complexed to a cationic lipid formulation (32). Lung tissues were harvested $6 \mathrm{~d}$ after injection and stained for routine histologic evaluation. Multifocal perivascular accumulations of mononuclear cells were observed in multiple sections of lung tissues from SEA DNA-injected mice $(b)$. By contrast, inflammatory changes were not observed in lung tissues of mice injected with empty vector DNA $(a)$. At a higher magnification, the perivascular infiltrate was found to be comprised primarily of mononuclear cells $(c, d$, and $e)$ and in some cases formed typical granulomas $(d)$. Immunostaining of lung sections with an anti-CD4 antibody revealed numerous CD4+ T cells in the perivascular infiltrate $(f)$, whereas CD4 + T cells were not increased in lung tissues of empty vector DNA-injected mice (not shown). Numerous Mac- $1+$ cells were also present in the perivascular infiltrates, along with some CD8+ T cells (not shown). $\times 85(a$ and $b)$, $\times 170(c$ and $d)$, and $\times 300(e$ and $f)$. 


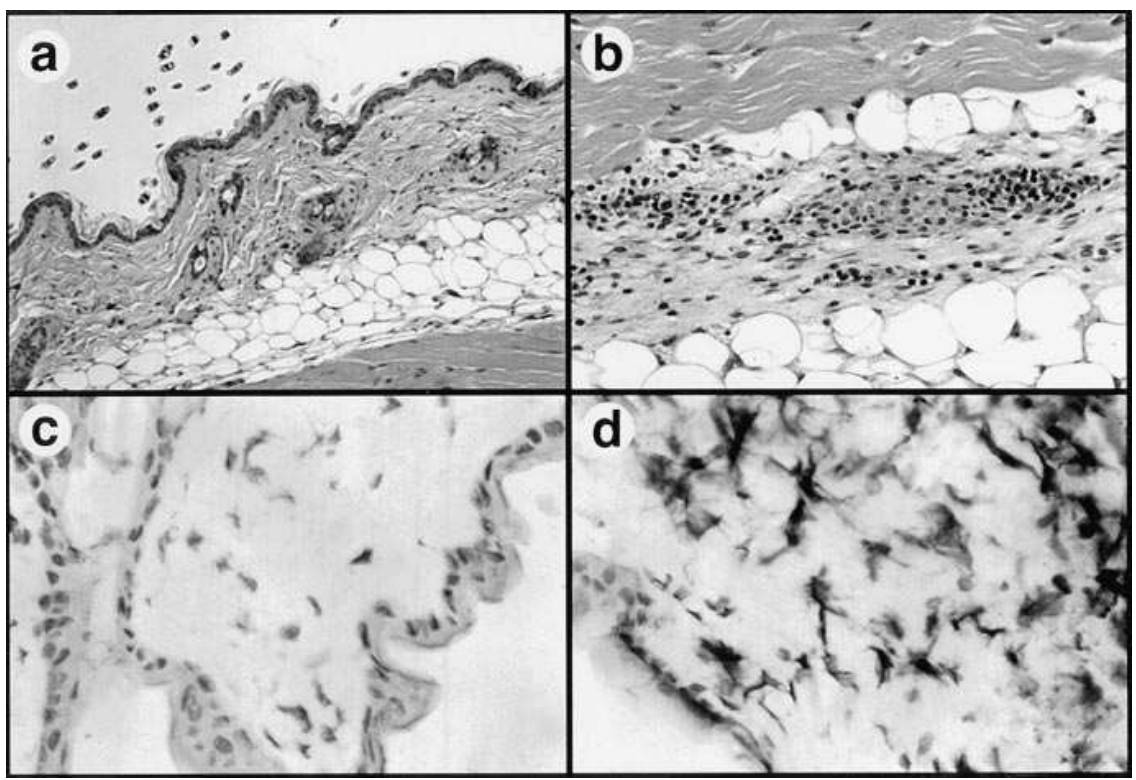

Figure 6. Intradermal SAg DNA injection induces epidermal infiltration of CD4+ T cells. Mice were injected intradermally in flank skin with either $50 \mu \mathrm{g}$ SEA DNA or $50 \mu \mathrm{g}$ empty vector DNA (mock injection). $6 \mathrm{~d}$ later, skin tissues were harvested, fixed, and stained with hematoxylin and eosin. A mild mononuclear cell infiltrate was present in subdermal tissues of SEA-injected mice $(b)$, but not in mice injected intradermally with empty vector DNA (a). Immunohistochemical staining of similarly treated tissues with an antibody to CD4 identified a diffuse infiltrate of CD4+ T cells in the epidermis of SEA-injected mice (dark staining cells, $d$ ), but not in mice injected with empty vector DNA $(c)$. Similar changes were observed in skin tissues from three different SEA injected mice. trophils) were also observed in the cellular infiltrate. The T cells probably arrived in muscle tissues by emigration from blood vessels, as suggested by the presence of numerous mononuclear cells adjacent to intramuscular blood vessels at early time points after injection (Fig. $5 \mathrm{f}$ ). We have also observed local proliferation of T cells in SAg DNA-injected muscle tissues (Dow, S.W., unpublished data). Thus, tissue expression of SAg genes serves as a stimulus for both $\mathrm{T}$ cell and monocyte immigration and local proliferation.

Cutaneous inflammatory responses induced by intradermal injection of $S A g D N A$. Previous studies have determined that plasmid DNAs can also be expressed efficiently after intradermal injection (36). We therefore assessed the response of cutaneous tissues to intradermal injection of plasmid DNA-encoding SEA. Injection of SEA DNA, but not empty vector DNA, induced a mononuclear inflammatory response in dermal tissues (Fig. 6, $a$ and $b$ ), though the magnitude of the response was less than in muscle tissues. In addition, a pronounced infiltrate of CD4 $+\mathrm{T}$ cells was observed in epidermal tissues of SEA DNA-injected mice, but not in empty vector-injected mice (Fig. $6 c$ and $d$ ). Infiltration of Mac- $1+$ cells was also observed in epidermal and dermal tissues (data not shown). The $\mathrm{CD} 8+\mathrm{T}$ cell response was less in skin than in muscle tissues (data not shown).

Intravenous injection of SAg DNA induces intrapulmonary inflammation. Previous studies have demonstrated intrapulmonary expression of reporter genes after intravenous injection of plasmid DNA complexed to liposomes $(32,37,38)$. We therefore investigated the pulmonary inflammatory response to intrapulmonary expression of SAg genes. Lung tissues from mice injected intravenously with SEA plasmid DNA complexed to cationic lipids contained numerous foci of mononuclear cells in a perivascular orientation (Fig. 7, $b-e$ ), consistent with endothelial cell SEA gene expression. In most sections, the cellular composition of these foci resembled granulomas (e.g., Fig. $7 d$ ). By immunohistochemistry, the foci were found to be comprised primarily of CD4+ T cells (Fig. $7 f$ ) and macrophages (not shown). Injection of empty vector DNA and lipids induced only minimal pulmonary inflammation (Fig. 7 a). Despite the presence of multifocal pulmonary infiltrates in SEA DNA-injected mice, the mice did not exhibit obvious respiratory distress, and repeated intravenous SEA DNA injections did not induce detectable illness (data not shown). Thus, intrapulmonary expression of SAg genes elicited a strong but apparently self-limiting mononuclear cell perivascular inflammatory response.

Mice injected with SAg DNA by any of three routes did not develop adverse effects, either locally in the muscles (lameness) or lungs (respiratory distress) or systemically (anorexia, weight loss). Mice observed for up to 6 mo after intramuscular SAg DNA injection remained healthy (data not shown). Thus, SAg gene expression in a variety of tissues induced local mononuclear cell inflammatory responses without inducing overt toxicity.

\section{Discussion}

Numerous studies have established that bacterial SAgs are potent, TCR-specific T cell activators (1-7). These properties of SAgs have been extremely valuable tools for investigating mechanisms underlying $\mathrm{T}$ cell activation, anergy, tolerance induction, and peripheral deletion. Superantigens are also attractive agents for use in immunotherapy. For certain applications, the ability to achieve sustained, locally expressed SAg activity has distinct advantages over systemic administration of SAg proteins, both in terms of more specific immune modulation and reduced toxicity. We therefore used a genetic therapy approach to address two aims: $(a)$ to evaluate functional SAg expression in mammalian cells in vitro and $(b)$ to assess the tissue inflammatory response to SAg gene expression in several different tissues in vivo.

The data presented here demonstrate that three different bacterial SAg genes (SEA, SEB, and TSST-1) were capable of expressing biologically active proteins in a variety of different mammalian cells when placed under the control of a strong eukaryotic promoter. Superantigen proteins present in both supernatants and lysates of transfected cells stimulated proliferation of human and mouse lymphocytes. In addition, SAgs 
expressed by mammalian cells retained their specificity for TCR V $\beta$ chains, indicating that any posttranslational modifications of the SAg protein made by mammalian cells did not alter either SAg function or specificity.

Mammalian cells produced transient high concentrations of SAg proteins intracellularly, as demonstrated by intense staining of intracellular deposits of SEB and SEA protein in transfected cells (Fig. 3). High concentrations of intracellular SAgs, however, may induce cellular cytotoxicity, as evidenced by the inability to detect the same high levels of SAg gene expression in stably transfected cell lines. A previous study also demonstrated expression of the TSST- 1 gene intracellularly in yeast (39). We have also expressed several other staphylococcal SAg genes in mammalian cells (Dow, S., unpublished data). These results suggest that most bacterial SAgs can be expressed in mammalian cells using eukaryotic expression vectors.

Bacterial superantigens were constituitively secreted by in vitro transfected cells at levels more than sufficient for $\mathrm{T}$ cell activation (20). The mechanism underlying the movement of SAg proteins across intact cell membranes is currently unknown, but could represent either active transport or passive diffusion along a concentration gradient. Studies with mutated SAgs may determine those portions of the SAg molecules responsible for transcellular movement.

Histologic evaluation of three different SAg DNA-injected tissues indicated that localized expression of SAg genes served as a potent stimulus for mononuclear cell infiltration. Local, sustained SAg expression by SAg gene transfer was necessary to induce the prolonged mononuclear cell inflammatory response. For example, direct intramuscular injection of recombinant SEA induced only a minimal inflammatory reaction, compared to intramuscular expression of SEA DNA (Fig. 5). Intradermal injection or percutaneous application of SEB protein was reported recently to induce localized cutaneous inflammatory responses in mice (40). The response consisted initially of Langerhan's cell activation, vasodilation, and neutrophil accumulation, followed in $48 \mathrm{~h}$ by mononuclear cell infiltration, which then resolved over 4-5 d (40). A similar response was observed in cutaneous tissues of mice in our study, except that the duration of the response was prolonged compared to the inflammatory response that was reported to have been induced by injection of SAg protein.

The perivascular location of the inflammatory responses observed in lung tissues of SEA DNA-injected mice is consistent with endothelial cell transfection and local gene expression. Lesions were not observed histologically in other tissues of SEA DNA-injected mice (kidney, brain, spleen, heart), except for mild periportal mononuclear cell infiltrates in hepatic tissues (data not shown). These data are consistent with the results of previous reporter gene studies using DOTIM-cholesterol lipids, which demonstrated preferential gene expression in pulmonary tissues (32). Pulmonary inflammatory lesions were also not observed in mice injected intravenously with SEA protein (data not shown), further emphasizing the importance of local SAg gene expression in mediating the inflammatory response.

The cellular response to SAg gene expression was similar in all three tissues examined. Approximately $50 \%$ of the cellular infiltrate consisted of $\mathrm{T}$ cells, with CD4+ $\mathrm{T}$ cells being more numerous than CD8+ T cells, especially in skin and lung tissues. Particularly in the epidermis of SEA DNA-injected skin, the infiltrate consisted almost entirely of CD4+ cells
(Fig. 6). Mac-1+ cells (monocytes) were also abundant in SAg-induced inflammatory foci, and were particularly numerous in muscle tissues. In all three tissues examined, the lesion induced by SAg gene expression was characteristic of a delayed-type hypersensitivity response. Thus, local SAg expression may be useful for inducing TH1-type $\mathrm{T}$ cell responses in tissues, using either direct DNA injection techniques or lipidmediated gene targeting.

The use of SAg gene expression to induce localized inflammatory responses has obvious application to the treatment of cancer. It was reported previously that intratumoral injections of plasmid DNA encoding an allogeneic MHC molecule, which also induces local inflammatory reactions, could slow tumor growth experimentally (41). We have found that repeated direct injections of SEB DNA plus cytokine DNA into spontaneous malignant melanomas in dogs induce a pronounced inflammatory cell infiltrate, followed in many cases by tumor regression, development of systemic antitumor immune responses, and prolonged survival (Dow, S.W., Elmslie, R., and Potter, T.A., manuscript submitted for publication). From a safety standpoint, direct injection of plasmid DNA avoids problems associated with viral vectors, including the dangers of transfection of germ line cells or acquisition of viral replication competence. In addition, the extreme potency of SAgs makes it possible to induce biologic effects with even low in vivo transfection efficiencies.

In summary, we have shown that bacterial SAgs can be expressed efficiently by mammalian cells in vitro and in vivo. In vivo expression of SAg genes in several different tissues induced a prominent $\mathrm{T}$ cell infiltrate, and did not induce toxicity or irreversible local pathology. Thus, direct injection and local expression of bacterial SAg genes may be useful for a variety of immunotherapeutic applications.

\section{Acknowledgments}

We thank Kathy Morgan for excellent secretarial assistance, and Andrew Willson for excellent technical assistance. We also thank Drs. Cori Gorman and Lisa Roche at the Megabios Corporation for providing the lipid-DNA complexes used for intravenous injection studies.

This work was supported in part by Public Health Service grant AI952-05 and by a grant from the Colorado Cancer League. T. Potter is a scholar of the Leukemia Society of America.

\section{References}

1. Peavy, D.L., W.H. Adler, and R.T. Smith. 1970. The mitogenic effects of endotoxin and staphylococcal enterotoxin B on mouse spleen cells and human peripheral blood lymphocytes. J. Immunol. 105:1453-1458.

2. Langford, M.P., G.J. Stanton, and H.M. Johnson. 1978. Infect. Immun. 22:62-68.

3. Herman, A., J.W. Kappler, P. Marrack, and A.M. Pullen. 1991. Superantigens: mechanism of T-cell stimulation and role in immune responses. Annu. Rev. Immunol. 9:745-772.

4. Ochi, A., K. Yuh, K. Migita, and Y. Kawabe. Effects of Staphylococcal toxins on T cell activity in vivo. 1992. Chem. Immunol. 55:115-136.

5. Kotzin, B.L., D.Y.M. Leung, J. Kappler, and P. Marrack. 1993. Superantigens and their potential role in human disease. Adv. Immunol. 54:99-165.

6. Marrack, P., and J. Kappler. 1990. The staphylococcal superantigens and their relatives. Science (Wash. DC). 248:1066.

7. Micusan, V.V., and J. Thibodeau. 1993. Superantigens of microbial origin. Semin. Immunol. 5:3-11.

8. Jardetzky, T.S., J.H. Brown, J.C. Gorga, L. Stern, R.G. Urban, Y. Chi, C. Stauffacher, J.L. Strominger, and D.C. Wiley. 1994. Three dimensional structure of a human class II histocompatibility molecule complexed with superantigen. Nature (Lond.). 368:711-718.

9. Choi, Y., A. Herman, D. DiGusto, T. Wade, P. Marrack, and J. Kappler. 
1990. Residues of the variable region of the T cell receptor B-chain that interact with $S$. aureus superantigens. Nature (Lond.). 346:471-473.

10. Gasciogne, N.R., and K.T. Ames. 1991. Direct binding of secreted T-cell receptor to superantigen associated with class II major histocompatibility complex protein. Proc. Natl. Acad. Sci. USA. 88:613-616.

11. White, J., A. Herman, A. M. Pullen, R. Kubo, J. Kappler, and P. Marrack. 1989. The V $\beta$-specific superantigen staphylococcal enterotoxin B: stimulation of mature T cells and clonal deletion in neonatal mice. Cell. 56:27-35.

12. Fleischer, B., and H. Schrezenmeier. 1988. T cell stimulation by staphylococcal enterotoxins. Clonally variable response and requirement for major histocompatibility complex class II molecules on accessory target cells. J. Exp. Med. 167:1697-1707.

13. Fraser, J.D. 1989. High affinity binding of staphylococcal enterotoxins A and B to HLA-DR. Nature (Lond.). 339:221-223.

14. Scholl, P.R., A. Diez, R. Karr, R.P. Sekaly, J. Trowsdale, and R.S. Geha. 1990. Effect of isotypes and allelic polymorphism on the binding of staphylococcal enterotoxins to MHC class II molecules. J. Immunol. 144:226-230.

15. Herman, A., G. Croteau, R.P. Sekaly, J. Kappler, and P. Marrack. 1990. HLA-DR alleles differ in their ability to present staphylococcal superantigens to T cells. J. Exp. Med. 172:709-717.

16. Janeway, C.A., J. Yagi, P.J. Conrad, M.E. Katz, B. Jones, S. Vroegop, and S. Buxser. 1989. T-cell responses to Mls and to bacterial proteins that mimic its behavior. Immunol. Rev. 107:61-88.

17. Choi, Y., B. Kotzin, L. Herron, J. Callahan, P. Marrack, and J. Kappler. 1989. Interaction of Staphylococcus aureus toxin "superantigen" with human T cells. Proc. Natl. Acad. Sci. USA. 86:8941-8945.

18. Herman, A., N. Labrecque, J. Thibodeau, P. Marrack, and J. Kappler. 1991. Identification of the staphylococcal enterotoxin A superantigen binding site in the b-1 domain of the human histocompatibility antigen HLA-DR. Proc. Natl. Acad. Sci. USA. 88:9954-9958.

19. Panina-Bordignon, P., X.T. Fu, A. Lanzavecchia, and R.W. Karr. 1992. Identification of HLA-DR alpha chain residues critical for binding of the toxic shock syndrome toxin superantigen. J. Exp. Med. 176:1779-1784.

20. Carlsson, R., H. Fischer, and H.O. Sjogren. 1988. Binding of staphylococcal enterotoxin A to accessory cells is a requirement for its ability to activate human T cells. J. Immunol. 140:2484-2488.

21. Rott, O., H. Wekerle, and B. Fleischer. 1992. Protection from experimental allergic encephalitis by application of a bacterial superantigen. Int. Immunol. 4:347-353.

22. Kawamura, T., M. Nagata, T. Utsugi, and J. Yoon. 1993. Prevention of autoimmune type I diabetes by CD4+ suppressor $\mathrm{T}$ cells in superantigentreated non-obese diabetic mice. J. Immunol. 151:4362-4370.

23. Gelfand, E.W., J. Saloga, and G. Lack. 1995. Modification of immediate hypersensitivity responses by staphylococcal enterotoxin B. J. Clin. Immunol. 15:37S-41S.

24. Schwab, J.H., R.R. Brown, S.K. Anderle, and P.M. Schlievert. 1993. Superantigen can reactivate bacterial cell wall-induced arthritis. J. Immunol. 150: 4151-4159.

25. Schiffenbauer, J., H.M. Johnson, E.J. Butfiloski, L. Wegrzyn, and J.M. Soos. 1993. Staphylococcal enterotoxins can reactivate experimental allergic encephalomyelitis. Proc. Natl. Acad. Sci. USA. 90:8543-8546.
26. Newell, K.A., J.D.I. Ellenhorn, D.S. Bruce, and J.A. Bluestone. 1991. In vivo T-cell activation by staphylococcal enterotoxin B prevents outgrowth of a malignant tumor. Proc. Natl. Acad. Sci. USA. 88:1074-1078.

27. Ochi, A., K. Migita, J. Xu, and K. Simonovitch. 1993. In vivo tumor immunotherapy by a bacterial superantigen. J. Immunol. 151:3180-3186.

28. Dohlsten, M. L., P. Abrahamsen, P. Bjork, P.A. Lando, G. Hedlund, G. Forsberg, T. Brodin, N.J.R. Gascoigne, C. Forberg, P. Lind, and T. Kalland. 1994. Monoclonal antibody-superantigen fusion proteins: tumor specific agents for T-cell-based tumor therapy. Proc. Natl. Acad. Sci. USA. 91:8945-8949.

29. Murray, D.L., D.H. Ohlendorf, and P.M. Schlievert. 1995. Staphylococcal and streptococcal superantigens: their role in human diseases. ASM News. 61:229-235.

30. Azevedo, J.C.S. 1989. Animal models for toxic shock syndrome: an overview. Rev. Infect. Dis. 11:S205-S209.

31. Miethke, T., C. Wahl, K. Heeg, B. Echtenacher, P.H. Krammer, and H. Wagner. 1992. T cell mediated lethal shock triggered in mice by the superantigen staphylococcal enterotoxin B: critical role of tumor necrosis factor. J. Exp. Med .175:91-98.

32. Solodin, I., C.S. Brown, M.S. Bruno, C. Chow, E. Jang, R.J. Debs, and T.D. Heath. 1995. A novel series of imidazloinium compounds for in vitro and in vivo gene delivery. Biochemistry. 34:13537-13544.

33. Zlotnik, A., R.P. Shimonkevitz, M.L. Gefter, J. Kappler, and P. Marrack. 1983. Characterization of the gamma interferon mediated induction of an tigen presenting activity of P388D1 cells. J. Immunol. 131:2814-2820.

34. Hamad, A.R.A., A. Herman, P. Marrack, and J.W. Kappler. 1994. Monoclonal antibodies defining functional sites on the toxin superantigen staphylococcal enterotoxin B. J. Exp. Med. 180:615-621.

35. Ulmer, J.B., J.J. Donnelly, S.E. Parker, G.H. Rhodes, P.L. Felgner, V.J. Dwarki, S.H. Gromkowski, R.R. Deck, C.M. DeWitt, A. Friedman, et al. 1993. Heterologous protection against influenza by injection of DNA encoding a viral protein. Science (Wash. DC). 259:1745-1749.

36. Raz, E., D.A. Carson, S.E. Parker, T.B. Parr, A.M. Abai, G. Aichiniger, S.H. Gromkowski, M. Singh, D. Lew, M.A. Yankauckas, et al. 1994. Intradermal gene immunization: the possible role of DNA uptake in the induction of cellular immunity to viruses. Proc. Natl. Acad. Sci. USA. 91:9519-9523.

37. Thierry, A.R., Y. Lunardi-Iskandar, J.L. Bryant, P. Rabinovich, R.C Gallo, and L.C. Mahan. 1995. Systemic gene therapy: biodistribution and longterm expression of a transgene in mice. Proc. Natl. Acad. Sci. USA. 92:97429746.

38. Zhu, N., D. Liggitt, Y. Liu, and R. Debs. 1993. Systemic gene expression after intravenous DNA delivery into adult mice. Science (Wash. DC). 261:209-211.

39. Deresiewicz, R.L., J.A. Falxenburg, M. Chan, R.W. Finberg, and D.L. Kasper. 1994. Intracellular expression of toxic shock syndrome toxin 1 in Saccharomyces cerevisiae. Infect. Immun. 62:2202-2207.

40. Saloga, J., D.Y.M. Leung, C. Raerdon, R.C. Giorno, W. Born, and E.W. Gelfand. 1996. Cutaneous exposure to the superantigen staphylococcal enterotoxin B elicits a T-cell dependent inflammatory response. J. Invest. Dermatol. 106:982-988.

41. Plautz, G.E., Z. Yang, B. Wu, X. Goa, L. Huang, and G. Nabel. 1993. Immunotherapy of malignancy by in vivo gene transfer into tumors. Proc. Natl. Acad. Sci. USA. 90:4645-4649. 